\title{
Immunohistochemistry for p53 is a useful tool to identify cases of acute myeloid leukemia with myelodysplasia-related changes that are TP53 mutated, have complex karyotype, and have poor prognosis
}

\author{
Sebastian Fernandez-Pol, Lisa Ma, Robert S Ohgami and Daniel A Arber \\ Department of Pathology, Stanford University, Stanford, CA, USA
}

\begin{abstract}
In this study, we evaluate the expression of p53 in core biopsies with acute myeloid leukemia and correlate the level of expression with acute myeloid leukemia subtype, TP53 mutation status, karyotype, and survival. Of the 143 cases evaluated, 71 fulfilled the WHO 2016 criteria for acute myeloid leukemia with myelodysplasia-related changes, 40 were acute myeloid leukemia-not otherwise specified, 25 were acute myeloid leukemia with recurrent genetic abnormalities, and 7 were therapy-related acute myeloid leukemia. By immunohistochemistry, $17 \%$ showed p53 expression in $>5 \%$ of the cells. Of the 24 cases with $>5 \%$ p53-positive cells, 17 were acute myeloid leukemia with myelodysplasia-related changes, 5 were acute myeloid leukemia-not otherwise specified, 1 was acute myeloid leukemia with recurrent genetic abormalities, and 1 was therapy-related acute myeloid leukemia. In cases for which data was available, expression of $>5 \%$ p53-positive cells was significantly associated with genotype $(n=67)$ and/or karyotype $(n=130)$. Among the 115 cases for which clinical follow up was available, the overall survival of cases with p53 expression $>15 \%$ (Median $=102$ days) was significantly shorter compared with cases with 553 expression $\leq 15 \%$ (Median $=435$ days). Within the acute myeloid leukemia with myelodysplasia-related changes group, this association remained significant, with cases with $\leq 15 \%$ p53positive cells having a median overall survival of 405 days versus 102 days for cases with $>15 \%$ p53-positive cells. Among acute myeloid leukemia with myelodysplasia-related changes cases with a complex karyotype, the finding of $>15 \%$ p53-positive cells was significantly associated with worse overall survival. The poor prognosis associated with more than 15\% p53-positive cells was independent of age and karyotype. In acute myeloid leukemia with myelodysplasia-related changes, p53 expression may be useful to infer TP53 mutation status, complex karyotype, and/or poor prognosis in situations where other modalities are not readily available. Modern Pathology (2017) 30, 382-392; doi:10.1038/modpathol.2016.206; published online 9 December 2016
\end{abstract}

Among the subtypes of acute myeloid leukemia, acute myeloid leukemia with multilineage dysplasia represents a particularly aggressive subtype with a high mortality and poor response to treatment. ${ }^{1-3}$ Although acute myeloid leukemia with myelodysplasia-related changes is defined by particular clinical, morphologic, and cytogenetic features, there remains significant heterogeneity in clinical behavior and response to

Correspondence: Dr S Fernandez-Pol, MD, PhD, Department of Pathology, Stanford University, 300 Pasteur Drive, Lane 235, Stanford, CA 94305-5324, USA.

E-mail: sfernand@stanford.edu

Received 21 July 2016; revised 18 October 2016; accepted 19 October 2016; published online 9 December 2016 therapy among patients with this disease. For this reason, continued efforts to identify prognostic and predictive features are necessary to guide clinical decisions, including decisions regarding intensity of therapy.

The tumor suppressor TP53 is the most commonly mutated gene in human cancers ${ }^{4}$ and mutations in TP53 have been demonstrated in several studies to correlate with inferior overall survival among patients with acute myeloid leukemia and myelodysplastic syndrome. ${ }^{5-8}$ In addition, TP53 mutations are found in $\sim 70 \%$ of cases with a complex karyotype, while in cases with normal karyotype, mutations are comparatively rare. ${ }^{9}$ Although genotyping 
TP53 in clinical specimens has become relatively common, immunohistochemical staining for p53 has been shown to correlate with the presence of missense mutations in TP53 in several tumor types, and thus has some utility in identifying malignancies with TP53 mutations which have a worse prognosis. ${ }^{10,11}$ Expression of p53 in acute myeloid leukemia has been examined previously, ${ }^{12,13}$ but limited data are available regarding p53 expression in different World Health Organization acute myeloid leukemia subtypes. In therapy-related myeloid neoplasms, previous work has shown that overexpression of p53 correlates with TP53 mutation and independently predicts a decreased overall survival. ${ }^{14}$ In acute myeloid leukemia arising from myelodysplastic syndrome, p53 was found to be expressed in $\geq 5 \%$ of cells in 9 of 39 cases. ${ }^{15}$ In this same study, a p53 expression level of $\geq 5 \%$ did not stratify higher-risk myelodysplastic syndrome and secondary acute myeloid leukemia into groups with different overall survival. Further work is necessary to evaluate the utility of p53 immunohistochemistry in other acute myeloid leukemia subtypes.

In this study, we set out to correlate expression of p53 with acute myeloid leukemia subtype, karyotype, TP53 genotype, and survival in a cohort of 143 cases of acute myeloid leukemia. We find that cases of acute myeloid leukemia with a high level of p53 expression are typically acute myeloid leukemiamyelodysplasia-related changes as opposed to acute myeloid leukemia-not otherwise specified or acute myeloid leukemia with recurrent genetic abnormalities. In addition, cases with higher p53 expression frequently have TP53 mutations, complex karyotypes and show a worse overall survival. Our data suggest that p53 immunohistochemistry may provide useful prognostic information in cases of acute myeloid leukemia with myelodysplasia-related changes.

\section{Materials and methods}

\section{Case Selection and Immunohistochemistry}

We identified 143 cases of acute myeloid leukemia from the Stanford Department of Pathology (obtained from 12 May 2004 to 25 October 2013) for which the original diagnostic bone marrow core biopsy was available. Cases were classified according to the most recently available 2016 World Health Organization categories. ${ }^{1,3}$ After fixation in Bouin solution and decalcification in Formical-4 (StatLab, McKinney, TX, USA), sections of the paraffin-embedded core biopsy were stained with an antibody to p53 (clone DO-7 mouse, Ventana, 1:400 dilution, Ventana CC1 retrieval solution, pH 6.0). For the entire cohort, core biopsies were considered adequate for evaluation if there were at least $\sim 1000$ cells present for evaluation and this was the case even for biopsies with hypocellular marrow. This study was approved by Stanford University's institutional review board.

\section{Method for Quantifying p53 Expression}

Examination of the 143 cases revealed that the majority of the core biopsies showed only rare $(<1 \%)$ nuclei with dim to moderate expression of p53. These cases also tended to be those that lacked a detectable TP53 mutation (discussed in detail in the Results section). In contrast, a subset of cases, which tended to have nonsynomous TP53 mutations, showed a higher proportion of p53-positive nuclei with intensity ranging from dim to bright. Given that the background level of expression of p53 in TP53 WT cases was very low in terms of the proportion of positive cells as well as intensity, we reasoned that an increased proportion of even dimly positive cells was significant. For this reason, we chose to count dimly positive cells in all of the counts used in this study. We also think that counting every nucleus that is at least dimly positive may have the added benefit of reducing the interobserver variability compared with choosing a threshold at a moderate level of intensity. The p53-stained core biopsies were evaluated and the percent of all nucleated cells with at least 1+ positive nuclei (defined as above background as compared with negative cases) was determined by manual counting. In core biopsies with variability in p53 staining in different fields, hotspots were counted. After close evaluation, it was decided that precise quantification of p53 staining in cases in the $0-5 \% \quad(n=119)$ group could not be reliably subcategorized by manual counting without a high degree of interobserver variability. The cases in which p53 expression was greater than $5 \%$ were precisely quantified by taking pictures of two optical fields at 200x magnification, printing images of the fields and manually counting the number of p53positive nuclei. The average total number of cells counted per case was 1790 and the median was 1860 (range 1000-2925). For 15 cases with p53 score $>5 \%$, a second observer counted at least 1000 cells to determine the percent of cells expressing at least dim nuclear p53. A linear regression comparing the two observers resulted in a line with an $R^{2}=0.97$ (Supplementary Figure 1).

\section{TP53 Sequencing}

For 67 of the cases, unstained aspirate smears or frozen bone marrow samples were available to evaluate the TP53 gene by Sanger sequencing to detect the presence of known frequently occurring mutations. Targeted sequencing of the TP53 gene was performed as described previously ${ }^{16}$ with three primer sets: Set 1 Forward 5'-TCTGTCTCCTTCCTC TTCCTAC-3' and Reverse 5'-CTGCTCACCATCGCT ATCTG-3'; Set 2 Forward 5'-CACATGACGGAGGT TGTGAG-3' and Reverse 5'-TAGGGCACCACCACA CTAT-3'; Set 3 Forward 5'-CCTGATTTCCTTACT GCCTCTT-3' and Reverse 5'-TCTTGTCCTGCTTG CTTACC-3'. 


\section{Statistical Analysis}

Statistical tests used in this study are indicated in the text and in the figure legends and were performed using SPSS software (SPSS, Chicago, IL, USA) and/ or R software (https://cran.r-project.org/). Cox proportional hazard model was used for univariate and multivariate analyses for overall survival.

\section{Results}

\section{Patient Cohort}

A summary of cohort characteristics is provided in Table 1 . The average age of the study cohort was 55.9 years old (median 60, range 5-80) and consisted of $50 \%$ females. Of the 143 cases, 71 (50\%) fulfilled the WHO 2008 criteria for acute myeloid leukemia with myelodysplasia-related changes, $40 \quad(28 \%)$ were acute myeloid leukemia-not otherwise specified,
$25(18 \%)$ were acute myeloid leukemia with recurrent genetic abnormalities, and 7 (5\%) were therapyrelated acute myeloid leukemia.

\section{p53 Expression in 143 Cases of AML}

Of the 143 cases, 24 (17\%) contained more than 5\% of the cells in the marrow with nuclear p53 expression above background. Among the 119 cases that we categorized as having $\leq 5 \%$ p53-positive cells, cases either showed no definite p53-positive cells or at most fewer than $5 \%$ nuclei with very dim staining. Within each case with $>5 \%$ p53-positive nuclei, the staining intensity ranged from very dim to very bright. Examples of p53-stained core biopsies, together with relevant clinical information, are provided in Figure 1.

On the basis of the morphologic features, it appeared that the majority of the positive cells were blasts. In 5 cases, p53 expression was seen in

Table 1 Summary of cases evaluated in this study

\begin{tabular}{|c|c|c|c|c|c|c|}
\hline Case type & $\begin{array}{l}\text { Age (mean, } \\
\text { median, range) }\end{array}$ & Males (\%) & $\begin{array}{l}\text { Complex } \\
\text { karyotype }^{\mathrm{a}}\end{array}$ & TP53 mutation ${ }^{\mathrm{b}}$ & $\begin{array}{l}\text { Cases with }>5 \% \\
\text { p53-positive cells }\end{array}$ & $\begin{array}{l}\text { Cases with }>15 \% \\
\text { p53-positive cells }\end{array}$ \\
\hline $\begin{array}{l}\text { All cases } \\
n=143\end{array}$ & $\begin{array}{c}55.9 \mathrm{y} \\
60.0 \mathrm{y} \\
5-80\end{array}$ & $72(50.3 \%)$ & 28 of $130(21.5 \%)$ & $\begin{array}{l}12 \text { of } 67 \\
(17.9 \%)\end{array}$ & $24(17 \%)$ & $14(9.8 \%)$ \\
\hline $\begin{array}{l}\text { AML-MRC } \\
n=7149.7 \%\end{array}$ & $\begin{array}{l}56.7 \mathrm{y} \\
60 \mathrm{y} \\
11-76\end{array}$ & $40(56.3 \%)$ & 26 of $63(41.3 \%)$ & $\begin{array}{l}10 \text { of } 33 \\
(30.3 \%)\end{array}$ & $17(24 \%)$ & $11(16 \%)$ \\
\hline $\begin{array}{l}\text { AML-NOS } \\
n=4028.0 \%\end{array}$ & $\begin{array}{l}59.1 \mathrm{y} \\
61 \mathrm{y} \\
28-80\end{array}$ & $15(37.5 \%)$ & 0 of $36(0 \%)$ & 1 of $12(8.3 \%)$ & $5(13 \%)$ & $2(5 \%)$ \\
\hline $\begin{array}{l}\text { AML-RGA } \\
n=2517.5 \%\end{array}$ & $\begin{array}{l}46.3 \mathrm{y} \\
50 \mathrm{y} \\
5-78\end{array}$ & $13(52.0 \%)$ & 0 of $24(0 \%)$ & 0 of $15(0 \%)$ & $1(4 \%)$ & $0(0 \%)$ \\
\hline $\begin{array}{l}\text { AML-T } \\
n=74.9 \%\end{array}$ & $\begin{array}{l}63.3 \mathrm{y} \\
61 \mathrm{y} \\
55-76\end{array}$ & $4(57.1 \%)$ & 2 of $7(28.6 \%)$ & 1 of $7(14.3 \%)$ & $1(14 \%)$ & $1(14 \%)$ \\
\hline
\end{tabular}

Abbreviations: AML, acute myeloid leukemia; MRC, myelodysplasia-related changes; RGA, recurrent genetic abnormalities; T, therapy related. ${ }^{a}$ Karyotype data were available for 130 of the cases.

Figure 1 Representative core biopsies stained with p53 antibody. (a) Acute myeloid leukemia-not otherwise specified, TP53 not sequenced, $12.5 \%$ p53-positive cells by manual count. Patient alive at 1771 days. (b) Acute myeloid leukemia-not otherwise specified with normal karyotype, TP53 not sequenced, patient alive at 319 days, $4 \%$ p53-positive cells by manual count. (c) Acute myeloid leukemia with myelodysplasia-related changes with complex karyotype, TP53 not sequenced, $16 \%$ positive cells, patient deceased at 10 days. (d) Acute myeloid leukemia with myelodysplasia-related changes with complex karyotype, TP53 not sequenced, 19\% p53-positive cells, patient deceased at 102 days. (e) Acute myeloid leukemia with myelodysplasia-related changes with complex karyotype, TP53 not sequenced, $26 \%$ p53-positive cells, patient deceased at 247 days. (f) Acute myeloid leukemia with myelodysplasia-related changes with complex karyotype with TP53 mutation (C176F) detected, 79\% p53-positive cells, patient alive at 286 days. (g) Acute myeloid leukemia with myelodysplasia-related changes with complex karyotype, frameshift TP53 mutation, clinical follow up not available. (h) Acute myeloid leukemia with myelodysplasia-related changes (biphenotypic) with complex karyotype, only rare positive cells, no TP53 mutation detected, patient alive at 234 days. (i) Acute myeloid leukemia with myelodysplasia-related changes with complex karyotype, R273H TP53 mutation detected, 14\% p53-positive cells, clinical follow up not available. (j) Therapy-related acute myeloid leukemia with normal karyotype with rare strongly positive cells, no TP53 mutation detected, patient deceased at 189 days. (k) Acute myeloid leukemia with myelodysplasia-related changes normal karyotype with 11\% p53-positive cells, no TP53 mutation detected, clinical follow up not available. (l) Acute myeloid leukemia with myelodysplasia-related changes complex karyotype with rare dim positive cells, R196* TP53, patient deceased at 594 days. (m) Acute myeloid leukemia-not otherwise specified with WT TP53, alive at 423 days of follow up with $46 \%$ p53-positive cells. (n) Acute myeloid leukemia-not otherwise specified with TP53 V274F mutation and 7\% p53-positive cells. (o) Acute myeloid leukemia with recurrent genetic abnormalities with mutated NPM1, wild-type TP53, 15\% p53-positive cells. 
megakaryocyte nuclei (Supplementary Table 4). Four of these cases were acute myeloid leukemia with myelodysplasia-related changes and one was therapy-related acute myeloid leukemia. All of these cases had a complex karyotype and the proportion of p53-positive cells in the 5 cases ranged from 16 to $53 \%$. TP53 sequence data was available for the therapy-related acute myeloid leukemia case,

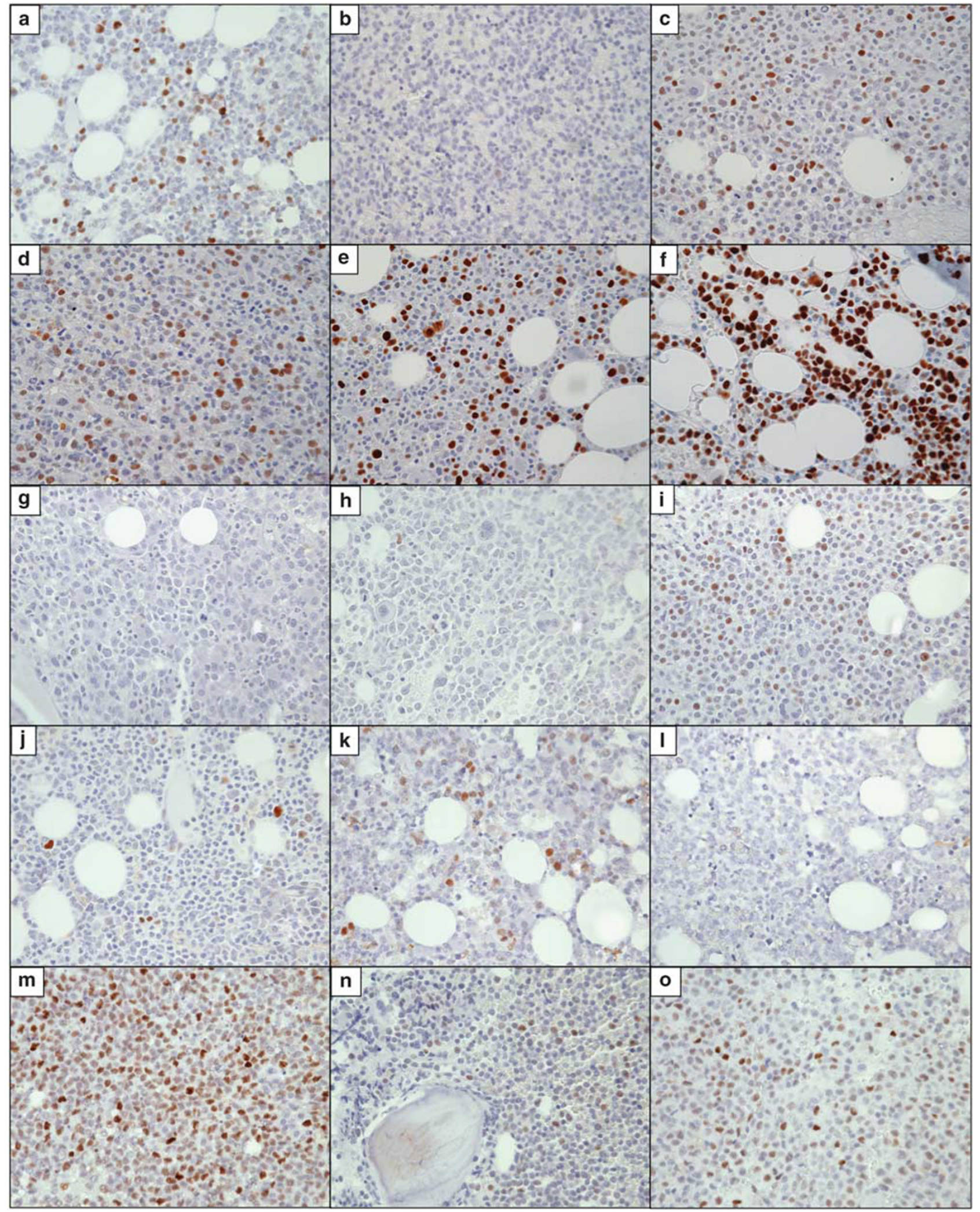




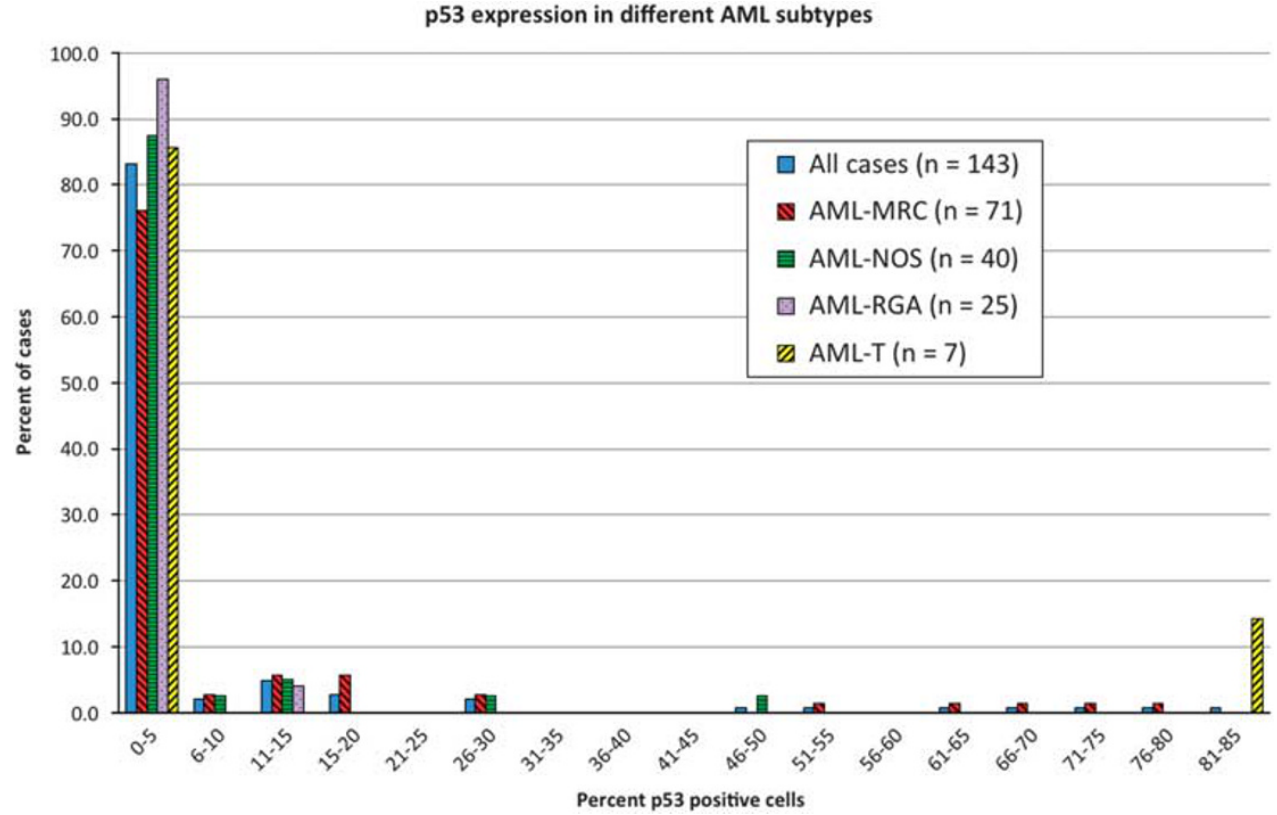

Figure 2 Percent of cases in each category with the indicated level of p53-positive cells. AML-MRC, acute myeloid leukemia with myelodysplasia-related changes, AML-NOS, acute myeloid leukemia-not otherwise specified, AML-RGA, acute myeloid leukemia with recurrent genetic abnormalities, AML-T, therapy-related acute myeloid leukemia.

which showed a mutation leading to an $\mathrm{R} 175 \mathrm{H}$ substitution.

On the basis of our evaluation of the stained biopsies, it appeared that distinction of a true p53 'null' phenotype, characterized by a complete absence of detectable p53 expression, was difficult to discern from cases with a very low level of p53 expression. In addition, our cohort contained only two cases in which the TP53 genotype might predicted to yield a p53 null phenotype due to loss of a significant portion of the WT protein. Both of these cases were acute myeloid leukemia with myelodysplasia-related changes and both showed very few p53-positive cells and included 1 case with a frameshift mutation (shown in Figure 1g) and 1 case with a nonsense mutation (shown in Figure 1l). Our conclusion was that it was difficult or impossible to reliably distinguish these cases from the numerous other cases with less than 5\% p53-positive cells that were TP53 WT.

Because of the difficulty in accurately quantifying p53 expression at low levels, we grouped cases with less than or equal to $5 \%$ p53-positive cells into one bin, which contained 119 of the cases. We grouped cases with $>5 \%$ expression into bins with intervals of $6-10 \%, 11-15 \%, 15-20 \%, 21-25 \%$, etc. Of the 24 cases with $>5 \%$ p53-positive cells, 17 were acute myeloid leukemia with myelodysplasia-related changes $(71 \%), 5$ were acute myeloid leukemia-not otherwise specified (21\%), 1 was therapy-related acute myeloid leukemia (4\%), and $1(4 \%)$ was acute myeloid leukemia-with recurrent genetic abnormalities. The distribution of p53 expression for all of the cases, as well as for each acute myeloid leukemia subcategory, is shown in Figure 2.

Five Cases of Acute Myeloid Leukemia-Not Otherwise Specified and 1 Case of Acute Myeloid Leukemia With Recurrent Genetic Abnormalities With Elevated p53 Expression

While cases with an elevated proportion of p53positive cells were more frequently seen in the acute myeloid leukemia with myelodysplasiarelated changes subcategory, 5 cases of acute myeloid leukemia-not otherwise specified and 1 case of acute myeloid leukemia with recurrent genetic abnormalities showed $>5 \%$ p53-positive nuclei. Details regarding these cases are provided in Table 2 and representative images of a subset of these cases are provided in Figure 1 (images a, n, and o). Thus, while an elevated proportion of p53-positive cells is found more frequently in acute myeloid leukemia with myelodysplasia-related changes (Table 1), our data does not support the notion that positive p53 immunohistochemistry can be used as a surrogate for acute myeloid leukemia with myelodysplasia-related changes.

\section{High p53 Expression Correlates With TP53 Mutation}

Evaluation of the 67 cases for which TP53 genotype data was available showed that cases with higher p53 expression appeared to be enriched for cases with non-synonymous mutations in TP53 (Figure 3a). There were two cases with TP53 mutations in which the proportion of p53-positive 
Table 2 Five cases of acute myeloid leukemia-not otherwise specified (AML-NOS) and one case of acute myeloid leukemia with recurrent genetic abnormalities (AML-RGA) with elevated p53 expression

\begin{tabular}{lcll}
\hline AML subtype & p53-positive cells & Follow up & TP53 status \\
\hline AML-NOS Figure 1a & $12.5 \%$ & Alive at 1771 days & Not available \\
AML-NOS Figure 1n & $7.1 \%$ & Not available & V274F \\
AML-NOS & $11 \%$ & Died at 31 days & Not available \\
AML-NOS & $27 \%$ & Died at 27 days & Not available \\
AML-NOS & $46 \%$ & Alive at 423 days & WT \\
AML-RGA (NPM1 mutated) Figure 1o & $15 \%$ & Not available & WT \\
\hline
\end{tabular}

a

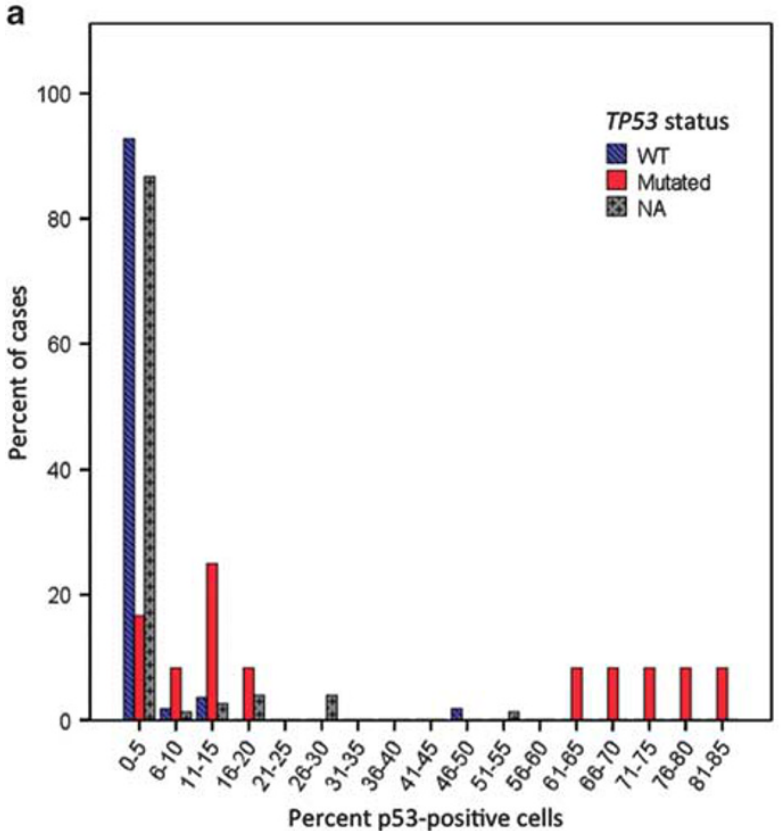

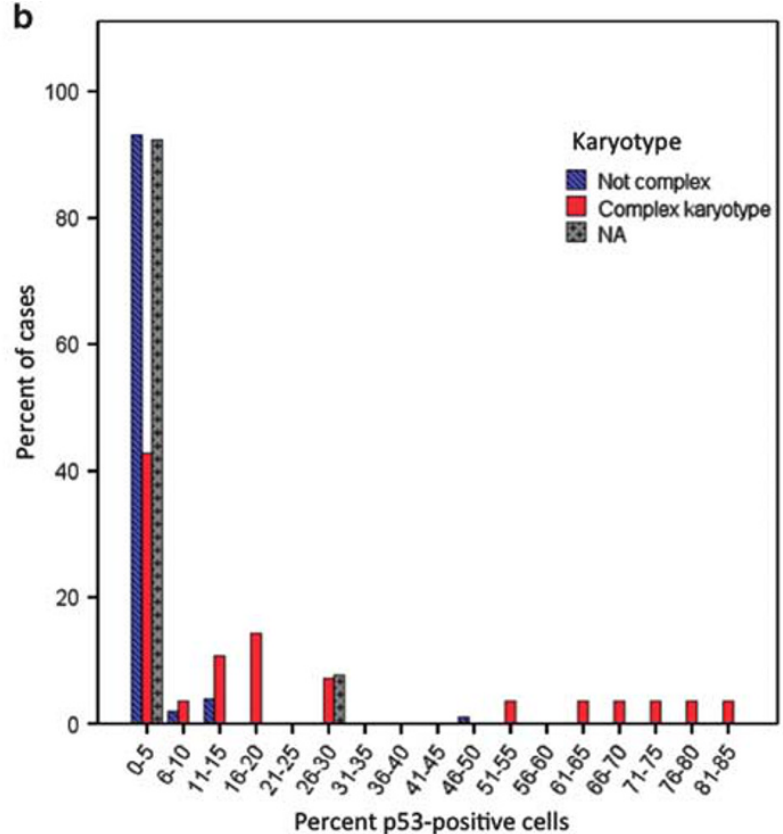

Figure 3 Distribution of p53 expression among cases for which TP53 was genotyped (a, $n=67)$ and for which karyotype data was available (b, $n=130$ ). In (a) and (b), the ${ }^{*}$ highlights one case of acute myeloid leukemia-not otherwise specified with normal karyotype from a patient who was alive at 423 days of follow up. WT, wild type. NA indicates cases for which TP53 was not sequenced (a) and cases for which karyotype was not available (b).

cells was $<5 \%$. One case had a frameshift mutation and another case had an R196* nonsense mutation (representative image of the latter case provided in Figure 1l). In examining these two cases, we could not identify a staining pattern that made these cases clearly discernible from the numerous wild-type TP53 cases with $<5 \%$ p53-positive cells.

Using the Fisher's exact test, a cutoff of $>5 \%$ was significantly associated with TP53 mutation $(P<0.0001)$. In order to evaluate the performance characteristics of utilizing p53 expression as a predictor for TP53 mutation, we constructed a receiver operating characteristic curve using different p53 expression levels and the results are shown in Table 3. The area under the curve when using p53 expression to detect TP53 mutations was 0.873 (95\% $\mathrm{CI}=0.745-1.000)$. Using a cutoff of $>5 \%$ p53positive cells resulted in a sensitivity of $83 \%$ and a specificity of $89 \%$ for detecting a TP53 mutation.
Using a cutoff of $>10 \%$ reduced the sensitivity to 0.75 and yielded an increase in specificity to $91 \%$.

As most of the cases that express p53 at $>5 \%$ in our study were acute myeloid leukemia with myelodysplasia-related changes, we performed a receiver operating characteristic curve analysis to evaluate the utility of using p53 expression for predicting a TP53 mutation in this subset of acute myeloid leukemias. As shown in Table 3, the area under the curve (0.844 95\% CI $=0.689-0.999)$ was similar to that seen when including all acute myeloid leukemia subtypes. The sensitivities and specificities using $>5,>10$, and $>15 \%$ cutoffs were also similar to those calculated when including all cases for which TP53 was genotyped. We conclude that p53 expression as determined by immunohistochemistry accurately predicts TP53 mutation status in acute myeloid leukemia when more than $5 \%$ of cells are positive. 
Table 3 Results of receiver operating characteristic curve for p53 expression and TP53 mutation

\begin{tabular}{|c|c|c|c|c|c|c|c|c|c|}
\hline & \multirow{2}{*}{ TP53 status } & \multirow{2}{*}{ Number of cases } & \multirow{2}{*}{$A U C$ 95\% CI } & \multicolumn{3}{|c|}{ Sensitivity } & \multicolumn{3}{|c|}{ Specificity } \\
\hline & & & & $>5 \%$ & $>10 \%$ & $>15 \%$ & $>5 \%$ & $>10 \%$ & $>15 \%$ \\
\hline \multirow[t]{2}{*}{ All cases } & WT & 55 & 0.873 & \multirow[t]{2}{*}{0.83} & \multirow[t]{2}{*}{0.75} & \multirow[t]{2}{*}{0.5} & \multirow[t]{2}{*}{0.89} & \multirow[t]{2}{*}{0.91} & \multirow[t]{2}{*}{0.94} \\
\hline & Mutated & 12 & $0.745-1.000$ & & & & & & \\
\hline \multirow[t]{2}{*}{ AML-MRC } & WT & 23 & 0.844 & \multirow[t]{2}{*}{0.8} & \multirow[t]{2}{*}{0.8} & \multirow[t]{2}{*}{0.5} & \multirow[t]{2}{*}{0.85} & \multirow[t]{2}{*}{0.89} & \multirow[t]{2}{*}{0.90} \\
\hline & Mutated & 10 & $0.689-0.999$ & & & & & & \\
\hline
\end{tabular}

Abbreviations: AML-MRC, acute myeloid leukemia with myelodysplasia-related changes, AUC, area under the curve.

\section{Association Between p53 Expression and Complex Karyotype}

In our cohort, information regarding karyotype was available for 130 cases, of which 102 (78\%) had a normal karyotype and $28(22 \%)$ had a complex karyotype. Of these 130 cases, 63 (48\%) were acute myeloid leukemia with myelodysplasia-related changes, 36 (28\%) were acute myeloid leukemianot otherwise specified, 24 (18\%) were acute myeloid leukemia with recurrent genetic abnormalities, and 7 (5\%) were therapy-related acute myeloid leukemia. To evaluate the association between p53 expression and karyotype, we examined the distribution of p53 expression and observed that cases with higher levels of p53 expression appeared to consist preferentially of cases with complex karyotype (Figure $3 b$ ). A cutoff of $>5 \%$ p53-positive cells was significantly associated with a complex karyotype $(P<0.0001$, Fisher's exact test). To evaluate the sensitivity and specificity of p53 immunohistochemistry for detecting cases with complex karyotype, we created an receiver operating characteristic curve using the two variables. Using all subtypes of acute myeloid leukemia, the calculated area under the curve was 0.761 (95\% CI=0.642-0.880) and cutoffs of $>5,>10$, and $>15 \%$ resulted in specificities of $>90 \%$ but low sensitivities for detecting cases with complex karyotype (Table 4). Restricting the analysis to cases of acute myeloid leukemia with myelodysplasia-related changes, and using the same p53 expression cutoffs, similarly showed specificities of $>90 \%$ but low sensitivities. We conclude that expression p53 in $>5 \%$ of cells in a bone marrow core biopsy involved by acute myeloid leukemia is not particularly sensitive but is highly specific for a complex karyotype.

\section{p53 Expression in Cases With Abnormalities of Chromosome 17}

As the TP53 gene resides on the short arm of chromosome 17 (17p13), ${ }^{17}$ we decided to evaluate whether p53 expression was related to cytogenetic abnormalities that included this region. Eleven of the 130 that were evaluated for cytogenetic abnormalities had -17 on karyotype. Of these 11 cases, 10 cases were acute myeloid leukemia with myelodysplasia-related changes and 1 case was therapy-related acute myeloid leukemia and 5 of the 11 cases had > 5\% p53-positive cells. TP53 was sequenced in 7 of the 11 cases, and 5 of these had a mutation in the gene, consistent with previous observations in other malignancies in which loss of one TP53 gene is often associated with mutation in the remain TP53 allele. ${ }^{18}$ The 2 cases that had a -17 and were TP53 WT were both acute myeloid leukemia with myelodysplasia-related changes with $\leq 5 \%$ p53-positive cells.

\section{p53 Expression is an Independent Poor Prognostic Factor}

In order to determine whether p53 expression correlates with prognosis, we obtained progressionfree survival and overall survival for 115 cases for which follow-up data were available. Evaluation of different cutoffs of p53 expression showed that there was no effect on progression free survival but that overall survival was significantly shorter in cases with $>5 \%$ p53 expression when examining all subcategories together as well as when restricting the analysis to cases of acute myeloid leukemia with myelodysplasia-related changes or simply acute myeloid leukemia with myelodysplasia-related changes cases with complex karyotype (Figure 4). We tested the effect of different cutoffs of p53 expression on overall survival and found that cutoffs between $>5 \%$ and $>20 \%$ stratified all acute myeloid leukemia cases into prognostically significant groups (Supplementary Table 1). These same cutoffs remained significantly associated with overall survival when limiting the survival analysis to the acute myeloid leukemia with myelodysplasia-related changes group (Supplementary Table 2). Within the group of acute myeloid leukemia with myelodysplasia-related changes with complex karyotype, cutoffs of $>5$ to $>15 \%$ stratified these cases into prognostically significant groups (Supplementary Table 3).

To determine if the prognostic impact of p53 expression was independent of the factors of age, karyotype, and TP53 genotype, we initially performed univariate regression analysis (Table 5). This analysis showed that age, karyotype, and p53 score (using a cutoff of $>15 \%$ ) were significantly 
Table 4 Results of receiver operating characteristic curve for p53 expression and karyotype

\begin{tabular}{|c|c|c|c|c|c|c|c|c|}
\hline \multirow{2}{*}{ Karyotype } & \multirow{2}{*}{ Number of cases } & \multirow{2}{*}{ AUC $95 \% C I$} & \multicolumn{3}{|c|}{ Sensitivity } & \multicolumn{3}{|c|}{ Specificity } \\
\hline & & & $>5 \%$ & $>10 \%$ & $>15 \%$ & $>5 \%$ & $>10 \%$ & $>15 \%$ \\
\hline \multicolumn{9}{|l|}{ All cases } \\
\hline Normal karyotype & 102 & 0.761 & 0.57 & 0.54 & 0.43 & 0.93 & 0.95 & 0.98 \\
\hline Complex karyotype & 28 & $0.642-0.880$ & & & & & & \\
\hline \multicolumn{9}{|l|}{$A M L-M R C$} \\
\hline Normal karyotype & 39 & 0.776 & 0.58 & 0.54 & 0.42 & 0.96 & 0.98 & 1 \\
\hline Complex karyotype & 26 & $0.650-0.903$ & & & & & & \\
\hline
\end{tabular}

Abbreviations: AML-MRC, acute myeloid leukemia with myelodysplasia-related changes, AUC, area under the curve.

a

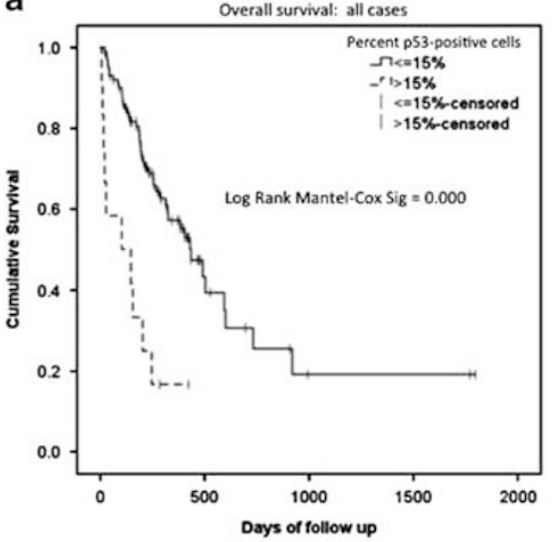

b

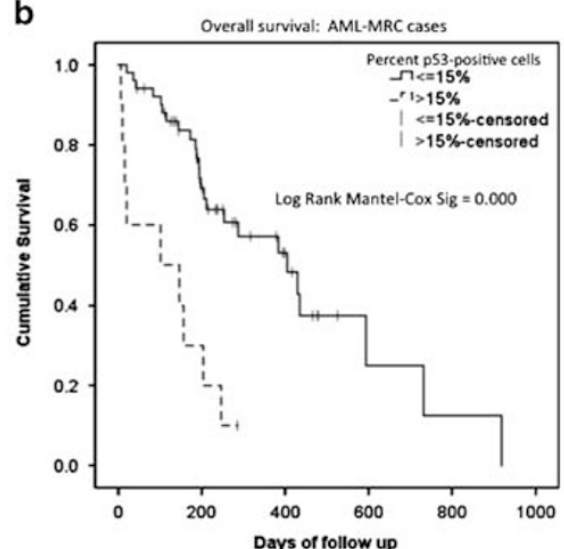

C

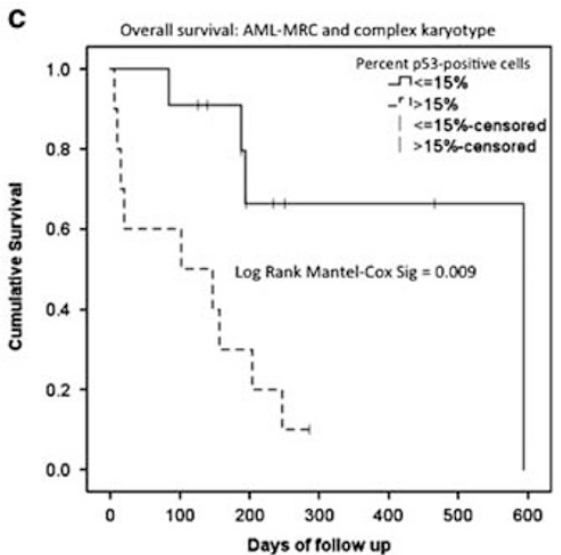

Figure 4 Overall survival in cases with $\leq 15 \%$ p53 expression compared with $>15 \%$ p53 expression in all cases of acute myeloid leukemia $(\mathbf{a}, n=115)$, cases of acute myeloid leukemia with myelodysplasia-related changes $(\mathbf{b}, n=61)$, and acute myeloid leukemiamyelodysplasia-related changes cases with complex karyotype $(\mathbf{c}, n=21)$. Kaplan-Meier curves and statistical tests were performed using IBM SPSS software. AML-MRC, acute myeloid leukemia with myelodysplasia-related changes.

Table 5 Univariate analyses for overall survival in all cases with clinical follow up

\begin{tabular}{lccl}
\hline All cases & Hazard ratio for death & 95\% confidence interval & P-value \\
\hline Age, $n=115$ & 1.03 & $1.01-1.05$ & 0.002 \\
TP5 mutation, $n=47$ & 2.43 & $0.80-7.4$ & 0.12 \\
Complex karyotype, $n=105$ & 2.51 & $1.33-4.74$ & 0.005 \\
p53 expression $>15 \%, n=87$ & 4.13 & $2.0-8.3$ & $7.9 \times 10^{-5}$
\end{tabular}

${ }^{\mathrm{a}}$ Wald test.

associated with survival. The lack of an association with survival with TP53 mutational status in our analysis is likely because only 47 of the cases that were genotyped for TP53 had data regarding overall survival. Subsequent multivariate analysis using age, karyotype, and p53 expression cutoff of $>15 \%$ showed that age and p53 expression were independently significantly associated with survival (Table 6).

A similar analysis using the variables of age, karyotype and p53 expression performed with only acute myeloid leukemia with myelodysplasia-related changes cases showed that age and p53 expression were associated with hazard of death in univariate analysis (Table 7). In a multivariate analysis using age and p53 expression, a p53 expression level of $>15 \%$ was associated with a hazard ratio (HR) for death of 4.7 (95\% CI 2.08-10.6; $P=0.0002$; Table 8). Age was also independently associated with death ( $\mathrm{HR}=1.03$, 95\% CI 1.003-1.06, $P=0.033$ ). As seen in the analysis performed using all cases, in the acute myeloid leukemia with myelodysplasia-related changes subgroup, the presence of TP53 mutation was not independently associated with an increased risk of death. The discrepancy between the association between TP53 mutation and p53 expression 
may be due to the fewer number of cases for which TP53 mutation and survival data were available ( $n=46$, with 20 events) compared with the number of cases for which data regarding p53 expression and survival data were available $(n=105$, with 48 events).

\section{Discussion}

Our findings largely agree with previous work showing that p53 is overexpressed in myeloid neoplasms with nonsynonymous TP53 mutations. ${ }^{19,20}$ Lepelley et $a l^{12}$ reported that 2 of 19 cases of acute myeloid leukemia demonstrated detectable p53 expression by immunohistochemistry and these cases also had missense TP53 mutations. In this study two other cases with no p53-positive cells had a nonsense mutation or a frameshift mutation in TP53. However, this study preceded subclassification of acute myeloid leukemia into more modern prognostically relevant subtypes. ${ }^{12}$ A later study by Zolota and colleagues evaluated p53 expression in 42 bone marrow biopsies classified according to FAB classification. ${ }^{13}$ In contrast to our findings, in their study, they found a higher proportion of cases with an elevated fraction of p53-postive nuclei. Specifically, $81 \%$ of the cases in their cohort showed $\geq 10 \%$ p53-positive nuclei. We speculate that this difference is due to the higher titer of DO-7 antibody used in their study (1:150) compared with the titer used in our study (1:400).

In contrast to the prior studies, our cohort is subclassified according to the 2016 WHO subgroups. Our study also included a relatively high percentage of acute myeloid leukemia with myelodysplasiarelated changes, possibly due to the predominance of older adults in the study, which is a particularly aggressive subtype of acute myeloid leukemia. To our knowledge, our study is the largest cohort of acute myeloid leukemia with myelodysplasia-related changes to be analyzed for p53 expression and correlated with TP53 mutation and survival. Another study evaluated p53 expression in acute myeloid leukemia evolving from a preceding myelodysplastic syndrome. ${ }^{15}$ In this report, 9 of 39 cases $(23 \%)$ of secondary acute myeloid leukemia showed strong nuclear staining (score $3+$ ) in at least $5 \%$ of the cells in the entire bone marrow. Their scoring strategy differed from ours in that they only counted strongly positive cells. The authors sequenced TP53 in 37 randomly selected bone marrow samples from their cohort of higher-risk myelodysplastic syndrome and secondary acute myeloid leukemia and found substantial agreement with p53 (Cohens Kappa $=0.645$ ). In lower risk myelodysplastic syndrome with del $(5 \mathrm{q})^{19}$ and in therapy-related myeloid neoplasms, ${ }^{20}$ a p53 cutoff of $\geq 1 \%$ was found to independently predict outcomes. Overall, there is likely interlaboratory variability in terms of p53 expression and thus any published cutoff for interpretation of
Table 6 Multivariate Cox proportional hazard model for overall survival in all cases with clinical follow up

\begin{tabular}{lccc}
\hline All cases $\mathrm{n}=115$ & $\begin{array}{c}\text { Hazard ratio } \\
\text { for death }\end{array}$ & $\begin{array}{c}\text { 95\% confidence } \\
\text { interval }\end{array}$ & P-value $^{a}$ \\
\hline Age & 1.04 & $1.01-1.06$ & 0.0016 \\
Karyotype & 1.50 & $0.64-3.51$ & 0.35 \\
p53 expression $>15 \%$ & 3.30 & $1.22-8.93$ & 0.019 \\
\hline
\end{tabular}

${ }^{\mathrm{a}}$ Wald test.

Table 7 Univariate analyses for overall survival in acute myeloid leukemia with myelodysplasia-related changes (AML-MRC) subgroup

\begin{tabular}{lccc}
\hline $\begin{array}{l}\text { AML-MRC } \\
\mathrm{n}=61\end{array}$ & $\begin{array}{c}\text { Hazard ratio } \\
\text { for death }\end{array}$ & $\begin{array}{c}\text { 95\% confidence } \\
\text { interval }\end{array}$ & P-value $^{a}$ \\
\hline $\begin{array}{l}\text { Age, } n=51 \\
\text { Complex karyotype, }\end{array}$ & 1.03 & $1.003-1.06$ & 0.033 \\
$\begin{array}{l}n=39 \\
\text { p53 expression }\end{array}$ & 1.78 & $0.85-3.74$ & 0.13 \\
$>15 \%, n=51$ & 4.70 & $2.08-10.6$ & 0.0002 \\
\hline
\end{tabular}

${ }^{\mathrm{a}}$ Wald test.

Table 8 Multivariate analyses for overall survival in acute myeloid leukemia with myelodysplasia-related changes (AMLMRC) subgroup

\begin{tabular}{lccc}
\hline $\begin{array}{l}\text { AML-MRC } \\
\mathrm{n}=61\end{array}$ & $\begin{array}{c}\text { Hazard ratio } \\
\text { for death }\end{array}$ & $\begin{array}{c}\text { 95\% confidence } \\
\text { interval }\end{array}$ & P-value $^{\mathrm{a}}$ \\
\hline $\begin{array}{l}\text { Age, } n=51 \\
\mathrm{p} 53 \text { expression }\end{array}$ & 1.03 & $1.003-1.07$ & 0.032 \\
$>15 \%, n=51$ & 4.75 & $2.09-10.8$ & 0.0002 \\
\hline
\end{tabular}

${ }^{\mathrm{a}}$ Wald test.

immunohistochemistry expression should be validated with cases known to have abnormal TP53. Furthermore, it is probably prudent to evaluate TP53 genotype in cases with elevated p53 expression.

Potential limitations of our study include our use of the proportion of nucleated cells expressing p53 in the marrow rather than the proportion of blasts expressing p53. However, it is likely that at least a subset of the non-blast cells in marrows with leukemia are derived from the neoplastic clone, have TP53 mutations, and consequently exhibit a p53-mutant immunophenotype. This proposal is supported by the cases in our cohort that had megakaryocytes with strong nuclear p53 staining and we are not the first group to report strong p53 expression in non-blast cells in cases of myelodysplastic syndrome with TP53 mutations. ${ }^{19,20}$ In addition, accurate quantification of the fraction of blasts expressing p53 would be challenging and would likely require staining with additional markers to 
accurately quantify blasts in the marrow. Thus, we do not see assessment of p53 expression as a proportion of all nucleated cells as a limitation. In any case, our study demonstrates that determining p53 expression as a fraction of all nucleated cells in the marrow is prognostically useful, predicts TP53 mutation, and is likely easily incorporated into clinical practice. It is also important to note, that, because our cohort contains relatively few cases with a high level of p53 expression and because we did not test cutoffs with a validation set, the cutoffs used here should be used as general guideline rather than as definitive values.

An additional limitation of our study is the approach used to sequence the TP53 gene. First, we did not sequence the entire TP53 gene, and thus there are possibly cases in which TP53 is mutated that were called TP53 WT in our study. As our sequencing targets known hotspots, the number of cases with mutations that were called WT is likely small, if indeed there are any. Despite these limitations, which would potentially result in an underestimate of the sensitivity and specificity of p53 immunohistochemistry to predict mutation status, our study suggests that p53 immunohistochemistry has a reasonable sensitivity and specificity for detecting TP53 mutations. In addition, the sensitivity and specificity of p53 immunohistochemistry for identifying TP53-mutated cases determined in our study are similar that seen in lung and ovarian tumors, ${ }^{17,21}$ suggesting that at least some of the limitations facing the use of immunohistochemistry in predicting TP53 abnormalities are shared across diverse neoplasms.

As sequencing of TP53 becomes routinely incorporated into sequencing panels used in the diagnostic workup of acute myeloid leukemia, it is reasonable to ask whether p53 immunohistochemistry would be redundant and unnecessary in most cases. Although our data suggest that p53 immunohistochemistry may be a useful ancillary test in cases of acute myeloid leukemia with myelodysplasiarelated changes to identify patients with a particularly poor prognosis, it is probably unnecessary to perform immunohistochemistry for p53 in all cases of acute myeloid leukemia. Because the turnaround time for immunohistochemistry is typically much shorter than molecular assays to assess TP53 genotype, it may be useful to perform p53 immunohistochemistry when a bone marrow biopsy shows features that are suspicious for dysplasia or in the case of patients with acute myeloid leukemia evolving from a preceding myelodysplastic syndrome. In addition, there are situations in which cytogenetic data is unavailable (eg, due to technical limitations, insufficient material, absent analyzable metaphases, etc) and in these cases, p53 immunohistochemistry may be useful to suggest whether a case of acute myeloid leukemia will be particularly aggressive.

\section{Acknowledgments}

We sincerely thank Li Ren for her essential contributions to the statistical analyses performed in the study.

\section{Disclosure/conflict of interest}

The authors declare no conflict of interest.

\section{References}

1 Swerdlow SH, Campo E, Harris NL, et al. (eds). WHO Classification of Tumours of Haematopoietic and Lymphoid Tissues, 4th edn. International Agency for Research on Cancer: Lyon, France, 2008, p 439.

2 Weinberg OK, Seetharam M, Ren L, et al. Clinical characterization of acute myeloid leukemia with myelodysplasia-related changes as defined by the 2008 WHO classification system. Blood 2009;113: 1906-1908.

3 Arber DA, Orazi A, Hasserjian R, et al. The 2016 revision to the World Health Organization (WHO) classification of myeloid neoplasms and acute leukemia. Blood 2016;127:2391-2405.

4 Levine AJ, Oren M. The first 30 years of p53: growing ever more complex. Nat Rev Cancer 2009;9:749-758.

5 Rücker FG, Schlenk RF, Bullinger L, et al. TP53 alterations in acute myeloid leukemia with complex karyotype correlate with specific copy number alterations, monosomal karyotype, and dismal outcome. Blood 2012;119:2114-2121.

6 Kihara R, Nagata Y, Kiyoi H, et al. Comprehensive analysis of genetic alterations and their prognostic impacts in adult acute myeloid leukemia patients. Leukemia 2014;28:1586-1595.

7 Ohgami RS, Ma L, Merker JD, et al. Next-generation sequencing of acute myeloid leukemia identifies the significance of TP53, U2AF1, ASXL1, and TET2 mutations. Mod Pathol 2015;28:706-714.

8 Robles AI, Harris CC. Clinical outcomes and correlates of TP53 mutations and cancer. Cold Spring Harb Perspect Biol 2010;2:a001016.

9 Haferlach C, Dicker F, Herholz H, et al. Mutations of the TP53 gene in acute myeloid leukemia are strongly associated with a complex aberrant karyotype. Leukemia 2008;22:1539-1541.

10 Bartek J, Iggo R, Gannon J, et al. Genetic and immunochemical analysis of mutant p53 in human breast cancer cell lines. Oncogene 1990;5:893-899.

11 Alsner J, Jensen V, Kyndi M, et al. A comparison between p53 accumulation determined by immunohistochemistry and TP53 mutations as prognostic variables in tumours from breast cancer patients. Acta Oncol 2008;47:600-607.

12 Lepelley P, Preudhomme C, Vanrumbeke M, et al. Detection of p53 mutations in hematological malignancies: comparison between immunocytochemistry and DNA analysis. Leukemia 1994;8:1342-1349.

13 Zolota V, Sirinian C, Melachrinou M, et al. Expression of the regulatory cell cycle proteins p21, p27, p14, p16, p53, mdm2, and cyclin $\mathrm{E}$ in bone marrow biopsies with acute myeloid leukemia. Correlation with patients' survival. Pathol Res Pract 2007;203:199-207. 
14 Cleven AH, Nardi V. Ok CY, et al. High p53 protein expression in therapy-related myeloid neoplasms is associated with adverse karyotype and poor outcome. Mod Pathol 2014;28:552-563.

15 Müller-Thomas C, Rudelius M, Rondak I-C, et al. Response to azacitidine is independent of p53 expression in higher-risk myelodysplastic syndromes and secondary acute myeloid leukemia. Haematologica 2014;99:e179-e181.

16 Ohgami RS, Ma L, Merker JD, et al. Next-generation sequencing of acute myeloid leukemia identifies the significance of TP53, U2AF1, ASXL1, and TET2 mutations. Mod Pathol 2015;28:706-714.

17 Mogi A, Kuwano H. TP53 Mutations in Nonsmall Cell Lung Cancer. J Biomed Biotechnol 2011;2011:1-9.
18 Gibbons DL, Byers LA, Kurie JM. Smoking, p53 Mutation, and Lung Cancer. Am Assoc Cancer Res 2014;12:3-13.

19 Saft L, Karimi M, Ghaderi M, et al. p53 protein expression independently predicts outcome in patients with lower-risk myelodysplastic syndromes with del (5q). Haematologica 2014;99:1041-1049.

20 Cleven AH, Nardi V, Ok CY, et al. High p53 protein expression in therapy-related myeloid neoplasms is associated with adverse karyotype and poor outcome. Mod Pathol 2015;28:552-563.

21 Mitsudomi T, Hamajima N, Ogawa M, et al. Prognostic significance of p53 alterations in patients with nonsmall cell lung cancer: a meta-analysis. Clin Cancer Res 2000;6:4055-4063.

Supplementary Information accompanies the paper on Modern Pathology website (http://www.nature.com/ modpathol) 